\title{
Circular RNA is enriched and stable in exosomes: a promising biomarker for cancer diagnosis
}

\author{
Cell Research (2015) 25:981-984. doi:10.1038/cr.2015.82; published online 3 July 2015
}

\section{Dear Editor,}

Circular RNAs (circRNAs) are recently identified as a naturally occurring family of widespread and diverse endogenous noncoding RNAs that may regulate gene expression in mammals [1-4]. They are unusually stable RNA molecules with cell type- or developmental stage-specific expression patterns [3,5]. Exosomes are small membrane vesicles of endocytic origin secreted by most cell types. They contain a specific cargo of protein, mRNA and microRNA species, which can modulate recipient cell behaviors and may be used as biomarkers for diagnosis of human diseases [6]. Here, we show for the first time the presence of abundant circRNAs in exosomes. RNA-seq analyses revealed that circRNAs were enriched in exosomes compared to the producer cells. Furthermore, the sorting of circRNAs to exosomes may be regulated by changes of associated miRNA levels in producer cells, and may transfer biological activity to recipient cells. Additionally, more than 1000 circRNAs were identified in human serum exosomes. circRNAs originated from human cancer xenografts could enter the circulation and be readily measured in the serum. Intriguingly, serum exosomal circRNAs were able to distinguish patients with colon cancer from healthy controls. Our results lay the foundation for development of circRNAs as a new class of exosome-based cancer biomarkers and suggest the potential biological function of exosomal circRNAs.

First we characterized circRNA transcripts by using RNA-seq analyses of ribosomal RNA-depleted total RNA from MHCC-LM3 liver cancer cells and cell-derived exosomes (Supplementary information, Figure S1). The prepared exosomes were examined by transmission electron microscopy (Supplementary information, Figure S2). A computational pipeline [3] based on anchor alignment of unmapped reads was used to identify circRNAs. Counts of reads mapping across an identified backsplice are normalized by read length and number of reads mapping (spliced reads per billion mapping (SRPBM)), which allows quantitative comparisons be- tween backsplices. Totally, 5484 and 6751 circRNAs were found in cells and exosomes, respectively (at least one backspliced read, SRPBM $>0.3$, Figure 1A). We refer to circRNA contained in exosomes as exo-circRNA. Exo-circRNAs were enriched by at least 2 -fold in exosomes compared to those in producer cells (Figure 1A; average of SRPBM: exo-circRNA, 1.32; cellular circRNA, 0.57). When using a stringent SRPBM cutoff, the number of exo-circRNAs is significantly higher than that of cellular circRNAs (Supplementary information, Figure S3; e.g., SRPBM $>1.2,1428$ of exo-circRNAs and only 319 of cellular circRNAs). We further validated this result by quantitative PCR (qPCR) analysis after reverse transcription (RT) of the same amount of cellular and exosomal RNAs, and confirmed the enrichment of five circRNAs (out of seven circRNAs) in exsomes (Supplementary information, Figure S4). The level of exo-circRNA was only moderately correlated with that of cellular circRNA (Figure 1B; Pearson correlation coefficient $r=0.43$ ), indicating that the sorting of specific circRNA species to exosomes may be actively regulated. The circRNAs identified from producer cells and exosomes and their correlations were confirmed by RNA-seq analysis of RNase R-treated samples (Supplementary information, Figure S5). We also purified cellular poly(A) RNA species and subjected them to RNA-seq analysis. Notably, we found that circRNAs were most abundant in exosomal RNA-seq library and also detectable in total cell RNA-seq library, whereas they were almost absent in poly(A) RNA-seq library (Supplementary information, Figure S3 and Figure 1C as an example). For each circRNA, we estimated the relative percentage of circRNAs to their linear counterparts by calculating the ratio of backsplice-spanning reads to reads spanning traditionally spliced junctions in both cells and exosomes. The result showed that the ratio of circRNA level to linear RNA level in exosomes was about 6-fold higher than that in cells (Figure 1D), indicating that circRNA was incorporated more into exosomes than linear RNA. We experimentally validated this result by qRT-PCR analysis of seven circRNAs (Supplementary information, Figure S6A). To 
A

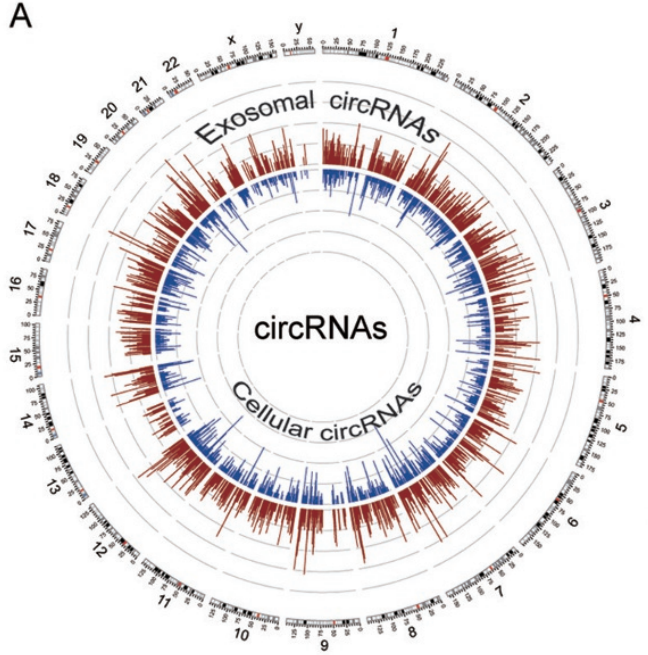

B

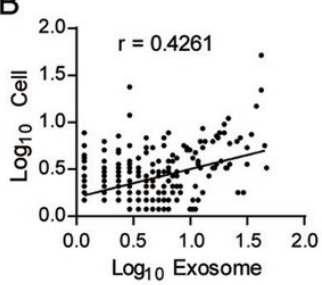

C

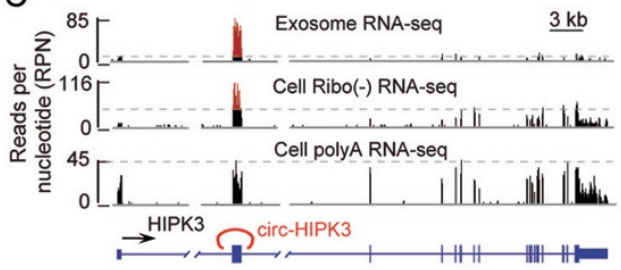

$\mathrm{F}$

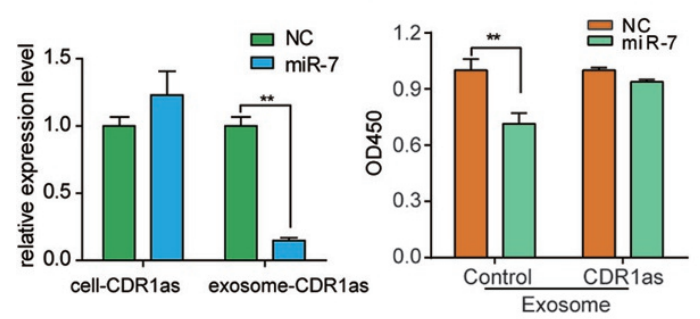

G

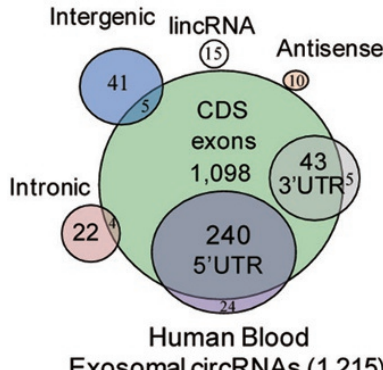

$\mathrm{K}$

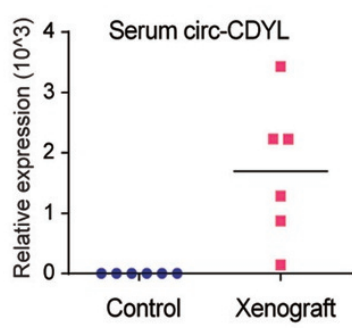

$\mathrm{H}$

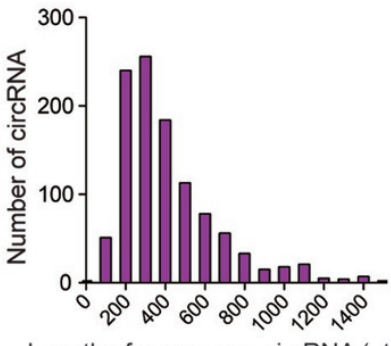

Length of serum exo-circRNA (nt)

L

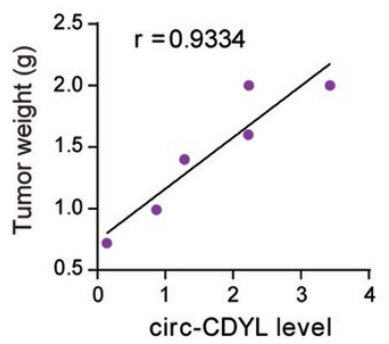

I
$J$
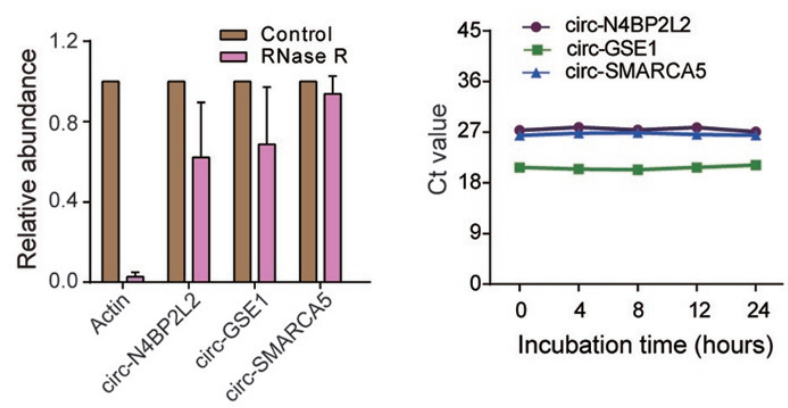

M

$\mathrm{N}$

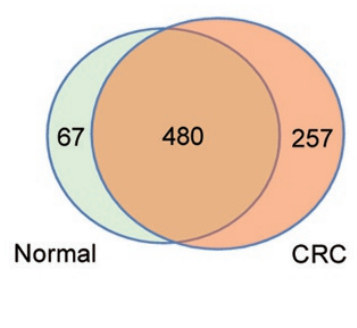

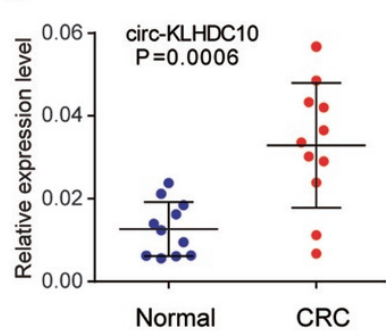

Figure 1 circRNAs are enriched and stable in exosomes and may serve as a potential biomarker for cancer detection. (A) Circos plots showing all circRNAs from cell and cell-derived exosome. (B) Correlation between cellular circRNA and exo-circRNA levels (SRPBM > 1). (C) Representative RNA-seq results for the HIPK3 gene and circ-HIPK3 (exon2) locus in various RNA-seq libraries. The read density of circ-HIPK3 was marked with red. (D) Comparison of the ratio of back-spliced to forward-spliced product reads between cell and cell-derived exosome. (E) qRT-PCR analysis of cellular CDR1as and exo-CDR1as circular RNA levels in cells and cell-derived exosomes after transfection with control or miR-7 mimics in HEK-293T cells. Data are presented as mean \pm SD. ${ }^{* *} P<$ 0.01 by Student's $t$-test. (F) Cell proliferation assay of SMCC-7721 cells after transfection with control or miR-7 mimics followed by treatment with control or CDR1as exosomes. CDR1as exosomes and control exosomes were collected from cell culture medium after transfection of CDR1as expression vector or control vector into HEK-293T cells. miR-7 mimics or negative control were transfected into SMCC-7721 cancer cells in 96-well plate. After $4 \mathrm{~h}$ transfection, CDR1as exosomes or control exosomes were added to the transfected cells. The cells were subsequently incubated for $48 \mathrm{~h}$, and cell proliferation was measured using the Cell Counting Kit-8 (CCK-8). Data are presented as mean \pm SD. ${ }^{* *} P<0.01$ by Student's $t$-test. (G) Genomic origin of human blood exo-circRNAs. (H) Distribution of serum exo-circRNA lengths ( $n=1107$, only exo-circRNAs with known spliced length were considered). (I) qRTPCR analysis of serum exo-circRNA levels after treatment with or without RNase R. The level without RNase R treatment was set as 1. Data are presented as mean $\pm S D$. (J) qRT-PCR analysis of serum exo-circRNA levels after incubation at room temperature for the indicated time. Ct values were shown. (K) Serum levels of exo-circCDYL were measured in all control and xenografted mice $(n$ $=6$, respectively). (L) Correlation between the level of tumor-derived exo-circCDYL in serum of xenografted mice and tumor mass. (M) Comparison of the exo-circRNA profile of normal serum and that of CRC serum. (N) qRT-PCR analysis of circ-KLDHC10 levels in normal serum and CRC serum ( $n=11$, respectively). 
further examine whether the presence and enrichment of circRNAs within exosomes is a common feature of cancer cells, we extended our analysis to a broader panel of cancer cell lines, including colon, lung, stomach, breast and cervical cancers. Indeed, we detected exo-circRNAs in all exosomes by qRT-PCR analysis (Supplementary information, Figure S6B).

circRNA has been reported to be able to bind to miRNA, which is also shown to be abundant in exosomes. This prompts us to investigate the relation of circRNA and miRNA, regarding the sorting of circRNA to exosomes. As CDR1as circRNA is known to act as a miR-7 sponge [1, 3], we introduced miR-7 mimics into cells and determined the level of CDR1as in cells and exosomes. Interestingly, we observed that the level of CDR1as circRNA was significantly downregulated in exosomes, but slightly increased in cells, upon ectopic expression of miR-7 in both HEK293T and MCF-7 cells (Figure 1E and Supplementary information, Figure S7). This result suggests that sorting of circRNAs to exosomes was regulated, at least in part, by changes of associated miRNA levels in producer cells. Furthermore, we found that exo-circRNA retained biological activity as the CDR1as exosomes could abrogate miR-7-induced growth suppression in receipt cells (Figure 1F).

Given that circRNAs are abundant in exosomes, we speculated that exo-circRNAs could be detected in the human blood. Then we characterized human circulating exo-circRNAs by RNA-seq analysis of exosomal RNAs from a pool of serum obtained from three healthy donors. With the SRPBM cutoff $>1.2$ (at least two backspliced reads), 1215 circRNAs were identified in the human serum exosomes (Supplementary information, Table S1). We annotated these circRNAs using the RefSeq database [7]. More than $90 \%$ of the circRNAs consist of protein-coding exons, whereas smaller fractions align to introns, lincRNA, unannotated regions of the genome and regions antisense to known transcripts (Figure 1G). The length of most serum exo-circRNAs was $<1000$ $\mathrm{nt}$, and the median length was only $350 \mathrm{nt}$ (Figure 1H). We further investigated the localization, integration and stability of circRNA in human serum. qRT-PCR analysis of three circRNAs revealed that circRNAs were predominantly located in serum-derived exosomes but not in exosome-depleted serum (Supplementary information, Figure S8). These circRNAs were resistant to digestion with RNase R exonuclease (Figure 1I), suggesting that exo-circRNAs remain to be circular and intact in exosomes. Exo-circRNAs were generally stable in serum as we found that incubation of the serum at room temperature for up to $24 \mathrm{~h}$ had minimal effect on circRNA levels (Figure 1J). The high stability of exo-circRNA might be due to the protection of exosomes or some specific sequence features and protein partners. Collectively, we showed that human serum exosomes contained a large number of intact and stable circRNAs.

To determine whether tumor-derived exo-circRNA enters the circulation and is measurable for cancer detection, we first employed a xenograft mouse model of human MHCC-LM3 cancer cells. Cells were subcutaneously implanted in the flank of nude mice. Serum was harvested 7 weeks later, and circulating exosomal RNAs were isolated for circRNA quantitation by qRT-PCR analysis. The human CDYL circRNA was able to be readily detected in the serum from tumor-bearing mice (Figure 1K). Abundance of tumor-derived exo-circRNA in serum of xenografted mice was correlated with tumor mass (Figure 1L). To strengthen the hypothesis that tumor-derived exo-circRNAs in the circulation could be used as a potential biomarker for cancer detection, we then explored the expression profile of serum exo-circRNAs in cancer patients and healthy donors. As done with normal serum (NS) circRNAs, the serum exosomes from 11 colorectal cancer (CRC) patients were pooled for RNA extraction prior to RNA-seq analysis. The expression profile of circRNAs in cancer serum was significantly different from that in NS. Compared to healthy subjects, 67 circRNAs were missing and 257 new circRNA species were detected in cancer patients (Figure 1M). Notably, many of the host genes for these new circRNAs (48 genes for 53 circRNAs) were significantly upregualted in CRC tissues (Supplementary information, Table S2). The RNA-seq results from pooled serum samples were further validated individually by qRT-PCR analysis. As shown in Figure 1N, expression levels of circ-KLDHC10 were significantly increased in cancer serum than those in NS. As the negative control, expression levels of circ-GSE1 were not different between cancer serum and NS (Supplementary information, Figure S9).

In conclusion, we demonstrated the existence of abundant exo-circRNAs, representing a novel class of stable RNA species in exosomes. Our finding that serum exo-circRNA may distinguish patients with cancer from healthy controls illustrates its significant translational potential as a circulating biomarker for cancer diagnosis. Furthermore, it would be of great interest to understand the biological functions of exo-circRNAs and find other potential applications in future studies.

\section{Acknowledgments}

We thank members of our laboratory for helpful discussion. We apologize to colleagues whose work could not be cited due to space constraints. Our work was supported by the National Natural 
Science Foundation of China (81472617 and 81125016), Shanghai Pujiang Program (14PJ1401900), and Shanghai Municipal Health Bureau (XYQ2011048 and XBR2011039).

\section{Yan $\mathrm{Li}^{1,{ }^{*}}$, Qiupeng Zheng ${ }^{1,2,{ }^{*}}$, Chunyang Bao ${ }^{1}$,} Shuyi $\mathrm{Li}^{1,2}$, Weijie Guo ${ }^{2}$, Jiang Zhao ${ }^{1}$, Di Chen ${ }^{2}$, Jianren $\mathrm{Gu}^{2}$, Xianghuo $\mathrm{He}^{1,2}$, Shenglin Huang ${ }^{1}$

${ }^{I}$ Department of Oncology, Shanghai Cancer Center and Institutes of Biomedical Sciences, Shanghai Medical College, Fudan University; Shanghai 200032, China; ${ }^{2}$ State Key Laboratory of Oncogenes and Related Genes, Shanghai Cancer Institute, Renji Hospital, Shanghai Jiao Tong University School of Medicine, Shanghai 200032, China

*These two authors contributed equally to this work.

Correspondence: Shenglin Huang ${ }^{\mathrm{a}}$, Xianghuo $\mathrm{He}^{\mathrm{b}}$

${ }^{\mathrm{a}}$ Tel: +86-21-3477-7580; Fax: +86-21-6417-2585
E-mail: slhuang@fudan.edu.cn

${ }^{b}$ E-mail: xhhe@fudan.edu.cn

\section{References}

1 Hansen TB, Jensen TI, Clausen BH, et al. Nature 2013; 495:384-388. Jeck WR, Sorrentino JA, Wang K, et al. RNA 2013; 19:141-157.

3 Memczak S, Jens M, Elefsinioti A, et al. Nature 2013; 495:333-338.

4 Salzman J, Gawad C, Wang PL, et al. PloS One 2012; 7:e30733.

5 Salzman J, Chen RE, Olsen MN, et al. PLoS Genet 2013; 9:e1003777.

6 Pant S, Hilton H, Burczynski ME. Biochem Pharmacol 2012; 83:14841494.

7 Pruitt KD, Tatusova T, Brown GR, et al. Nucleic Acids Res 2012; 40:D130-D135.

(Supplementary information is linked to the online version of the paper on the Cell Research website.) 